\title{
Intersubband Polaritons and Strong Coupling in Single Nanoantenna Observed by Near-field Microscopy
}

\author{
Chih-Feng Wang, ${ }^{1,2, *}$ Terefe G. Habteyes, ${ }^{2}$ Ting Shan Luk, ${ }^{3,4}$ John F. Klem, ${ }^{4}$ Igal Brener, ${ }^{3,4}$ \\ Hou-Tong Chen, ${ }^{1}$ and Oleg Mitrofanov ${ }^{3,5}$ \\ ${ }^{1}$ Center for Integrated Nanotechnologies, Los Alamos National Laboratory, Los Alamos, NM 87545, USA \\ ${ }^{2}$ Center for High Technology Materials, University of New Mexico, Albuquerque, NM 87106, USA \\ ${ }^{3}$ Center for Integrated Nanotechnologies, Sandia National Laboratories, Albuquerque, NM 87123, \\ ${ }^{4}$ Sandia National Laboratories, Albuquerque, NM 87185, USA \\ ${ }^{5}$ Electronic and Electrical Engineering, University College London, London, WC1E 7JE UK \\ * Present address: Physical Sciences Division, Pacific Northwest National Laboratory, Richland, WA 99354 \\ Authore-mail address: o.mitrofanov@ucl.ac.uk
}

\begin{abstract}
Strong coupling of intersubband excitation until recently was observed only in largescale systems. We demonstrate intersubband polariton formation in an isolated nanoantenna using near-field spectroscopy, enabling investigations of light-matter interaction in the single nanoantenna regime.

(C) 2020 The Authors.
\end{abstract}

\section{Introduction}

Intersubband (ISB) transitions in quantum wells (QWs) offer ample possibilities to explore and utilize light-matter interaction over a wide range of wavelengths, from near- to far-IR [1]. Plasmonic nanoantenna provided one route to achieve strong coupling and formation of polaritons [2], opening possibilities for interesting quantum effects, such as ISB polariton condensation [3], and proposals for a range of practical devices [4].

To explore polariton quantum phenomena, it is desirable to work with ISB polaritons in an isolated subwavelength-size system, for instance a single nanoscale antenna. For the first time, we reported observation of ISB polaritons in a single nanoantenna coupled to the ISB transition in QWs at $\sim 1000 \mathrm{~cm}^{-1}$ [5], and here we will discuss results of our investigations. Unlike several far-field attempts to reach the single-nanoantenna regime reported recently $[4,6]$, we use near-field microscopy and probe the light-matter coupling through the evanescent fields which exist on the metallic surface of an isolated resonator [7].

\section{Results}

We employ a commercial scattering-type scanning near-field microscopy system (neaSpec $\mathrm{GmbH}$ ) schematically illustrated in Fig. 1(a). Using short IR pulses $\left(\lambda_{c} \sim 10 \mu \mathrm{m}, 850-1300 \mathrm{~cm}^{-1}\right)$, we excite a nanoantenna (Fig. 1(b)) deposited on the surface of a stack of QWs. The near-field probe scatters a small fraction of the evanescent field, which carries the information about antenna interaction with the QWs underneath the surface. In order to analyze the evanescent field spectrally, we combine the scattered field with a reference IR pulse and perform coherent FTIR spectroscopy analysis.

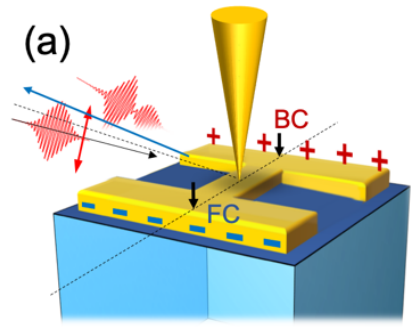

(b)

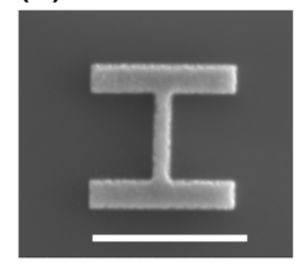

(c)

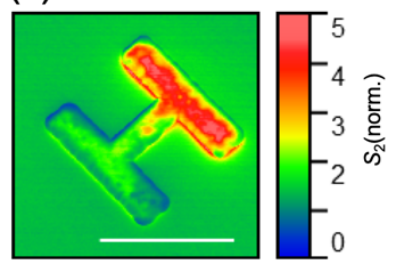

Fig. 1. (a) Schematic diagram of nano-FTIR spectroscopy of a metallic dogbone nanoantenna. The scattering tip is positioned at the center of the front (FC) or the back (BC) antenna bars (marked by black arrows). (b) SEM image of the $l=950 \mathrm{~nm}$ nanoantenna; the scale bar is $1 \mu \mathrm{m}$. (c) Evanescent field on the surface of nanoantenna excited by a CW IR laser light in resonance with the antenna frequency $\left(1000 \mathrm{~cm}^{-1}\right)$ [5]. 

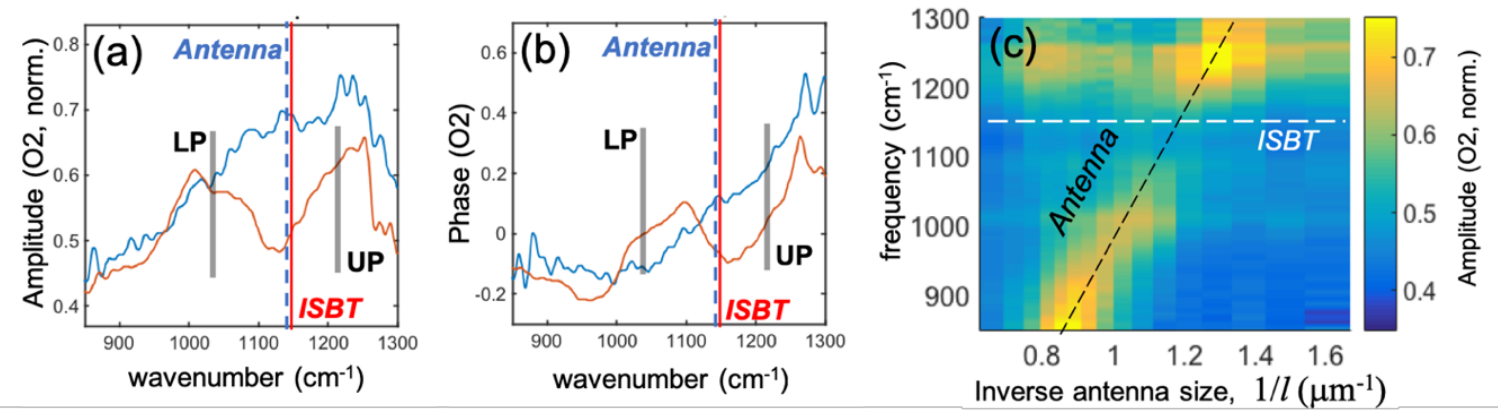

Fig. 2. Near-field FTIR amplitude (a) and phase (b) for the back bar of a nanoantenna $(l=850 \mathrm{~nm})$ in resonance with the ISBT transition $\left(1150 \mathrm{~cm}^{-1}\right)$ (red lines). The blue lines show amplitude and phase for a nanoantenna of the same size without coupling to the ISB transition. (c) Amplitude map for a range of nanoantennas with lengths $l$ from $600 \mathrm{~nm}$ to $1600 \mathrm{~nm}$. The antenna dispersion and the ISB transition are shown by the black and white dashed lines.

Results of nano-FTIR spectroscopy analysis for an antenna with the resonance at the frequency of the ISB transition $\left(1150 \mathrm{~cm}^{-1}\right)$ are shown in Fig. 2(a,b). Instead of the dipolar resonance peak we observe two peaks indicating the peak splitting into two distinct ISB polariton modes above and below the antenna frequency. For comparison, Fig. 2 also shows nano-FTIR spectra for a nanoantenna with the same dimensions, but fabricated on a sample with wider QWs, which exhibit the ISB transition at $815 \mathrm{~cm}^{-1}$. The observed splitting is indicative of strong coupling.

The nano-FTIR analysis also provides the phase of the evanescent fields on the surface. We find that in the case of two polariton peaks, the phase spectrum exhibits two step-like changes at the frequencies of the lower and upper polaritons, whereas the phase spectrum of the nanoantenna without ISB transition coupling exhibits only one step at the antenna resonance frequency. Detailed analysis of the phase spectra is discussed in Ref. 5.

We now map dispersion characteristics of the nanoantenna-ISB system in the single nanoantenna regime. We fabricate a set of nanoantennas with size $l$ ranging from $600 \mathrm{~nm}$ to $1600 \mathrm{~nm}$. It allows us to "tune" the dipolar resonance across the ISB transition and map the dispersion properties of the ISB polaritons. We find that the amplitude of the resonant antenna field is suppressed when the antenna and the ISB transitin are in resonance, and the nanoantennaISB system exhibits a mode anti-crossing behavior with the mode splitting of $\sim 190-200 \mathrm{~cm}^{-1}$.

\section{Conclusions}

We demonstrate that it is possible to investigate ISB polaritons in a single isolated nanoantenna regime in the IR frequency range, using nano-FTIR spectroscopy. This approach opens doors to a range of possible studies, e.g. investigations of the elusive effect of ISB polariton condensation. We anticipate that this near-field approach will also enable explorations of strong and ultrastrong light-matter coupling in the single nanoantenna regime for other excitations.

\section{Acknowledgements}

This work was supported by the U.S. Department of Energy, Office of Basic Energy Sciences, Division of Materials Sciences and Engineering. Fabrication and nano-FTIR measurements were performed at the Center for Integrated Nanotechnologies, an Office of Science User Facility operated for the U.S. Department of Energy (DOE) Office of Science. Sandia National Laboratories is a multi-mission laboratory managed and operated by National Technology and Engineering Solutions of Sandia, LLC., a wholly owned subsidiary of Honeywell International, Inc., for the U.S. Department of Energy's National Nuclear Security Administration under contract DE-NA-0003525. Los Alamos National Laboratory, an affirmative action equal opportunity employer, is managed by Triad National Security, LLC for the U.S. Department of Energy's NNSA, under contract $89233218 \mathrm{CNA} 000001$. This article describes objective technical results and analysis. The views expressed in the article do not necessarily represent the views of the U.S. DOE or the United States Government. C.-F. W and T. H. were supported in part by the U.S. National Science Foundation (Grant No. 1651478).

\section{References}

[1] A. F. Kockum, A. Miranowicz, S. De Liberato, S. Savasta and F. Nori, Nat. Rev. Phys. 1, 19-40 (2019).

[2] Y. Todorov and C. Sirtori, Phys. Rev. B 85, 045304 (2012).

[3] R. Colombelli and J.-M. Manceau, Phys. Rev X 5, 011031 (2015).

[4] A. Benz et al., Nat. Commun. 4, 2882 (2013).

[5] C.-F. Wang, T. G. Habteyes, T. S. Luk, J. F. Klem, I. Brener, H.-T. Chen, and O. Mitrofanov, Nano Lett. 19, 4620-4626 (2019).

[6] M. Malerba et al, Appl. Phys. Lett. 109, 021111 (2016).

[7] O. Mitrofanov, Y. Todorov, D. Gacemi, A. Mottaghizadeh, C. Sirtori, I. Brener, J.L. Reno, Opt. Express 26, 7437-7450 (2018). 